\title{
Effect of Educational Guidelines on Nurses' Knowledge and Practice Regarding Central Line Associated Blood Stream Infection at Intensive Care Unit
}

\author{
Fahema Yehia Mohamed Zeyada ${ }^{1}$,Seham A. Abd El-Hay', Heba A. Al-Metyazidy ${ }^{3}$ : \\ ${ }^{1}$ Nursing Specialist El santa Central Hospital \\ ${ }^{2}$ Assistant Prof. Medical- Surgical Nursing, Faculty of Nursing, Tanta University, Egypt. \\ ${ }^{3}$ Assistant Prof. Critical Care and Emergency Nursing, Tanta University, Egypt.
}

Abstract:

Background: Central line associated blood stream infections are serious but can often be prevented when evidence-based guidelines that are used by critical care nurse through utilized infection control measures during insertion and maintenance of central lines. Aim of the study was to evaluate the effect of educational guidelines on nurse's knowledge and practice regarding central line associated blood stream infection at intensive care unit. Subjects and Method: Research design: The present study utilized a quasi- experimental research design. Setting: The study was conducted at: Anesthesia Intensive Care Unit and Medical Intensive Care Unit of Emergency Hospital affiliated to Tanta Main University Hospital. Neurological Intensive Care Unit of Tanta Main University Hospital. The study subjects: All nurses (100) who are providing care for patients with central venous catheters and were having at least one year of experience. Tools: Two tools were used to collect the data; structured interviewing sheet and an observational checklist for nurses' practice. Results: The main results revealed that there was significant statistical improvement in the total knowledge and practice level among the studied nurses post nursing educational guidelines. Conclusion: the implementation of nursing educational guidelines has a positive impact not only in improving nurses' knowledge and practice regarding central line care but also, decrease rate of central line associated blood stream infection. Recommendation: it was recommended that nurses should attend in-service training programs for improving their knowledge and enhancing their practice.

Key words: Central Line Associated Blood Stream Infection, Educational Guidelines, Knowledge, Practice. 


\section{Introduction}

Central lines (CLs) have a multitude of complications that are associated with their placement. Health care associated infection particularly central line associated blood stream infection (CLABSI), contribute to the greatest threat to patient safety in critical care units which lead to increase mortality and morbidity rate. These complications can cause a significant healthcare burden in cost and patient quality of life. Recognition and management of central line complications are important when caring for patients with vascular access, but prevention is the ultimate goal $^{(1,2)}$.

The central line associated blood stream infection has been defined by the Centers for Disease Control and Prevention (CDC) as a laboratory-confirmed primary bloodstream infection in patients who had a CL for at least 48 hours, which is not related to an infection at another site. The use of CLs is common for critical ill patients. However, CLABSI is caused by microorganisms that colonize the external surface of the device or the fluid pathway when the device is inserted, as well as an infection that occurs over the course of use ${ }^{(3,4)}$. CLABSIs are serious but can often be prevented when evidence-based guidelines that are used by critical care nurse through utilized infection control measures during insertion and maintenance of CLs ${ }^{(5)}$. Following such guidelines which include optimal site selection that avoid femoral vein in adult patients and use of subclavian rather than jugular veins, the use of maximal sterile barrier precautions during catheter insertion, alcohol-based chlorhexidine $2 \%$ skin preparation and maximum barrier precautions ${ }^{(6)}$.

Additionally, maintenance guidelines consist of daily review of central line necessity, prompt removal of unnecessary lines, clean the hands with an alcoholbased hand rub solution before any manipulation of the infusion line, disinfect catheter hubs, ports, connectors before using central venous catheter (CVC) and removing any useless catheters ${ }^{(9,10)}$. Also, change dressings and disinfect site with alcohol-based chlorhexidine every 48hours and replace administration sets within 24 hours can considerably reduce the risk of infection and mortality in patients ${ }^{(11)}$.

The nurse play a major role in providing care to the patients in all phases of central line insertion to control the infection which can help for decreasing the risk of central venous catheter related blood stream infection ${ }^{(12)}$. They assist in CL insertion as they select the optimal site for insertion with avoidance of femoral veins in adult 
patients. The critical care nurse should maintain aseptic techniques, use maximal protective barrier precautions and use chlorhexidinegluconate $\quad 2 \% \quad$ skin preparation before insertion ${ }^{(13,14)}$.

After insertion, the role of the nurse not limited to giving intravenous antibiotic prophylaxis but also include care of dressing, withdrawing a venous blood sample, and delivery of drugs. As well, critical care nurses teach the patients and their family about the care of central venous catheters. Therefore, they contribute to the decline of infection. Besides, integrated infection control programs, including supervision of hospital acquired infections have brought to a notable decline in the rate of infections in the intensive care unit (ICU) with resulting lowered health care costs ${ }^{(15-18)}$.

\section{Significant of the study}

Critical care nurses have important roles in preventing CLABSI, so they must have the ability to know how to prevent complications associated with CL insertion and provide high quality of care. This is carried out through demonstrated of best practices utilization for assessment and maintenances of CVCs prior, during and at the end of the hospital duration which is the basic principles in preventing many complications related to CVC utilization (19). A well trained nursing team on assessment and maintenance of $\mathrm{CVC}$ and the application of standardized evidence based guidelines are key success factors strongly influencing the incidence of CVC long term complications ${ }^{(20)}$.

So, it is important for improving critical care nurses knowledge and practice which is the key factor in reducing CLABSI through educational guidelines that may result in significant decrease in critically ill patient's morbidity and mortality rate When staff nurses feel supported from their head nurses to suggest new processes and share their ideas in a supported environment, they will be more

\section{Aim of the Study}

Evaluate the effect of educational guidelines on nurse's knowledge and practice regarding central line associated blood stream infection at intensive care unit.

\section{Research hypothesis:}

Total level of knowledge and practice mean scores of intensive care unit nurses would be improved post implementation of educational guidelines regarding central line associated blood stream infection.

\section{Subjects and Method}

\section{Study design:}

The present study utilized a quasiexperimental research design which had been used to evaluate the effect of educational guidelines on nurse's 
knowledge and practice regarding central line associated blood stream infection at intensive care unit.

\section{Setting:}

The study was conducted at Anesthesia Intensive Care Unit and Medical Intensive Care Unit of Emergency Hospital affiliated to Tanta Main University Hospital. Neurological Intensive Care Unit of Tanta Main University Hospital.

\section{Subject:}

All nurses (100) from above mentioned settings who are providing care for patients with central venous catheters and were having at least one year of experience. They were divided as following: 50 nurses from Anesthesia Intensive Care Unit, 30 nurses from Medical Intensive Care Unit and 20 nurses from Neurological Intensive Care Unit.

\section{Tools of data collection}

Two tools were used to determine the effect of educational guidelines on nurse's knowledge and practice regarding central line associated blood stream infection, which include the following:-

\section{Tool (I): Structured interviewing:}

It was comprised of two parts:

\section{Part A: Socio demographic data of} nurses; which included; nurse's code, age, sex, marital status, level of education, occupation, years of experience and previous training about care of central line.

\section{Part B: Nurse's knowledge assessment} sheet:

It was developed by the researcher after reviewing of related literature ${ }^{(21-24)}$ to

evaluate nurses' knowledge pre, immediate and 2-months later post implementation of educational guidelines regarding central line associated blood stream infection.

\section{Scoring system of knowledge;}

Two level of scoring for questions were used as the following:

- Correct and complete answer will be scored (2)

- Correct and incomplete answer will be scored (1)

- Incorrect answer will be scored (0)

The total scoring system of nurses' knowledge was (112) and classified as the following:

-Good $\rightarrow \quad>75 \%$ of the total score

-Fair $\rightarrow \geq 60 \%-75 \%$ of the total score

-Poor $\rightarrow \quad<60 \%$ of the total score

Tool (II): An observational checklist regarding nurses' practice

This tool was developed by the researcher after reviewing relevant literatures ${ }^{(25-28)}$ to assess nurses' practice pre, during and 2months post implementation of educational guidelines about reducing CLABSI. It 
consisted of (146) subtitles classified under two main parts as the following:

Practice regarding central venous catheter procedure and practice regarding infection control measures.

\section{Scoring System of practice}

Two level of scoring for questions were used as the following:

-Done practice takes (1)

-Not done practice takes $(0)$

The total practices score will be (146) each right answer took one grade. The scoring system calculated (146) and classified as following:

-Satisfactory $\rightarrow \quad \geq 75 \%$ of the total score

-Unsatisfactory $\rightarrow<75 \%$ of the total score

\section{Method}

The study was accomplished through the following steps:

\section{1-Administrative process:}

a- Official permission from the faculty of nursing was delivered to the appropriate authorities at the two selected units to conduct the study.

b- Permission was obtained from the directors of:

- Anesthesia Intensive Care Unit and Medical Intensive Care Unit of Emergency Hospital affiliated to Tanta Main University Hospital.
- Neurological Intensive Care Unit of Tanta Main University Hospital.

\section{2-Ethical and legal considerations:}

a- Nature of the study did not cause any harm or pain to all subjects.

b- An informed consent was taken from every participant patient after complete explanation about the aim of the study.

c- Complete confidentiality and privacy was considered regarding data collection and results. A code number was used rather than names.

d- The nurse was informed the right to withdraw from the study at any time and without any reason.

\section{3-Tools development:}

All tools of the study were developed by the researcher to collect the data after extensive review of literature ${ }^{(30,20,29,30, \text { and 31). }}$.

4- Tools of data collection nurses' knowledge assessment sheet were translated into Arabic language.

\section{5- Validity of Tools:}

All tools of the study were reviewed for content validity and clarity by a panel of (5) expertise in the field of Medical Surgical Nursing, critical care nursing, anesthesia, medical and neurological field physicians. Their opinions were elicited regarding tools format and consistency, it was calculated and found to be $=(98 \%)$. 
6- Reliability: The reliability for the study tools was calculated by cronbach's alpha test, it was;

\section{- Cronbach's Alpha for first tool was}

0.761 for 64 items applied on 10 nurses.

- Cronbach's Alpha for second tool was 0.802 for 157 items applied on 10 nurses.

- Cronbach's Alpha for the sheet in total was $\mathbf{0 . 8 4 5}$ for 221 items applied on 10 nurses.

\section{7-A pilot study:}

It was conducted on (10) nurses to test the clarity, feasibility and the applicability of the different items of the determent tools to detect any obstacles during the period of data collection. The needed modification was done by the researcher before study according to the experience gained from this pilot study.

\section{8-Data collection:}

The subjects in pilot study are excluded from the current study. Data collection duration period was 6 months started from first of November 2019 to the end of May in 2020. The present study was conducted through four main phases (Assessment, planning, implementation and evaluation). Assessment phase: Assessment of the nurse baseline data that was carried out by using the following: Tool (I) part (A) was used to collect baseline data of nurses.
Tool (I) part (B) was used to assess nurses' knowledge regarding central line associated blood stream infection. Regarding tool (II), it was used to assess nurses' practice.

Planning phase: it included; preparation of the content of nursing educational guidelines and preparation of the environment.

Implementation Phase: The educational guidelines was conducted in (6) sessions to the nurses who will be divided into (5) groups, each group was contain (8) nurses three days per week and the time of each session was about 30 minutes.

The evaluation phase: Tool (I) part A was used to collect baseline data about nurses. Tool (I) part B and tool II were used to assess knowledge and practice pre, immediately and 2-months later post implementation of educational guidelines regarding CLABSI.

\section{Statistical analysis:}

The collected data were organized, tabulated and statistically analyzed using SPSS software statistical computer package version 26. For quantitative data, the range, mean and standard deviation were calculated. For qualitative data, comparison was done using Chi-square test $\left(\chi^{2}\right)$. For comparison between means of two variables in a group, paired samples ttest was used. For comparison between 
means for variables during three periods of intervention in a group, or for more than two variables, the F-value of analysis of variance (ANOVA) was calculated.

Correlation between variables was evaluated using Pearson and Spearman's correlation coefficient $r$. A significance was adopted at $\mathrm{P}<0.05$ for interpretation of results of tests of significance $(*)$. Also, a highly significance was adopted at $\mathrm{P}<0.01$ for interpretation of results of tests of significance $(* *){ }^{(32)}$.

\section{Results}

Table (1):shows the distribution of the nurses according to their sociodemographic characteristics. Concerning to age, this table revealed that more than half $(59.0 \%)$ of the studied nurses were aged from(21to31) years old, where nearly one-third $(31.0 \%)$ of them were in the age group from 31 to less than 40 years old, with mean age $(31.53 \pm 5.08)$.

Regarding to sex and marital status, the majority (86.0 \% and $80.0 \%)$ of the studied nurses were females and married. In relation to educational level and occupation, it was seen that nearly half $(49.0 \%)$ of the studied nurses had bachelor of nursing. Moreover nearly three-quarters $(71.0 \%)$ of the studied nurses were nursing staff, while more than one-quarter(29\%) of them were head nurses. In addition to; it was found that nearly half $(45.0 \%)$ of the studied nurses had $\geq 10$ years of nursing experience. According to Previous training about central line related blood stream infection, nearly four-fifths of the studied nurses (79.0\%) did not have previous training about central line related blood stream infection.

Figure (1): The figure showed that there was a highly statistical significant improvement in the total level of nurses' knowledge regarding CVC where more than three-quarters $(79 \%)$ and $(77 \%)$ of the studied nurses had poor and fair level of knowledge pre and post 2 months of nursing educational guidelines respectively, whereas all of them were good immediately after implementation of nursing educational guidelines.

Table (2): illustrates the distribution of the studied nurses according to their knowledge domains regarding CVC throughout all periods of implementation of nursing educational guidelines.

It was observed that there were highly statistical significant among the studied nurses regarding total knowledge mean score pre, immediately and post 2 months after implementation of nursing educational guidelines at $\mathrm{p}$ value $=0.000^{*}$.

Table (3):illustrates the distribution of the studied nurses according to their main practice domains levels throughout all 
periods of implementation of nursing educational guidelines.

The table revealed that there was statistical significant improvement in the total level of nurses practice regarding both $\mathrm{CVC}$ and infection control measures pre, immediately and post 2 months after implementation in nursing educational guidelines at $\mathrm{P}$ value $=0.000^{*}$

Table (4): illustrates the correlation between total knowledge domains and total practice domains among the studied nurses throughout all periods of implementation of nursing educational guidelines.

\section{Regarding pre nursing educational} guidelines, the table shows that there was positive significant correlation between total knowledge score \&total practice score $\mathrm{r}=0.227, \mathrm{P}=0.023$.

In relation to immediately after implementation of nursing e educational guidelines, the table shows that there was positive non-significant correlation between total knowledge score \&total practice score $\quad(\mathrm{r}=0.149, \quad \mathrm{P}=0.14)$ respectively.

While post 2 months of implementation of nursing educational guidelines, the table shows that there was positive nonsignificant correlation between total knowledge score \&total practice score $(\mathrm{r}=0.052 \mathrm{P}=0.605)$ respectively.
Table (5):demonstrates the correlation between socio-demographic characteristics and total knowledge score among the studied nurses throughout all periods of implementation of nursing educational guidelines.

It was noticed that there was a highly negative significant correlation between occupation of the studied nurses and total knowledge score pre educational guidelines as $(\mathrm{r}=-0.341, \mathrm{P}=0.001 * *)$ while immediately and 2 months after implementing educational guidelines there was non-significant correlation between occupation of the studied nurses and total knowledge score.

On the other hand there was nonsignificant correlation between age, gender and experience of the studied nurses and total knowledge score pre, immediately $\& 2$ months post nursing educational guidelines as $p>0.05$ respectively.

Table (6): demonstrates the correlation between socio-demographic characteristics and total practice score among the studied nurses throughout all periods of implementation of nursing educational guidelines.

It was noticed that there was negative significant correlation between age of the studied nurses and total practice score immediately after implementation of nursing educational guidelines $(\mathrm{r}=-0.213$, 
$\mathrm{P}=0.033)$, in contrary with pre and post 2 months of implementation of nursing educational guidelines there were negative non-significant correlation between age of the studied nurses and total practice score $\mathrm{P}>0.05$ respectively.

Also, there was non-significant correlation between gender, occupation and experience of the studied nurses and total practice score pre \&immediately after nursing educational guidelines $\mathrm{P}>0.05$ respectively. 
Table (1): Distribution of the studied nurses according to their Socio-demographic characteristics

\begin{tabular}{|c|c|c|}
\hline \multirow[t]{2}{*}{ Characteristics } & \multicolumn{2}{|c|}{$\begin{array}{l}\text { The studied nurses } \\
(\mathrm{n}=100)\end{array}$} \\
\hline & no & $\%$ \\
\hline $\begin{array}{l}\text { Age (in years) } \\
\text { - } 21-<31 \\
\text { - } 31-<40 \\
\text { - } 40-50\end{array}$ & $\begin{array}{l}59 \\
31 \\
10\end{array}$ & $\begin{array}{l}59.0 \\
31.0 \\
10.0\end{array}$ \\
\hline $\begin{array}{c}\text { Range } \\
\text { Mean } \pm \text { SD }\end{array}$ & \multicolumn{2}{|c|}{$\begin{array}{c}(23-46) \\
31.53 \pm 5.08 \\
\end{array}$} \\
\hline $\begin{array}{l}\text { Gender } \\
\text { - Male } \\
\text { - Female }\end{array}$ & $\begin{array}{l}14 \\
86\end{array}$ & $\begin{array}{l}14.0 \\
86.0\end{array}$ \\
\hline $\begin{array}{l}\text { Marital status } \\
\text { - Single } \\
\text { - } \text { Married }\end{array}$ & $\begin{array}{l}20 \\
80\end{array}$ & $\begin{array}{l}20.0 \\
80.0\end{array}$ \\
\hline $\begin{array}{l}\text { Educational level } \\
\text { - Technical institute } \\
\text { - Bachelor } \\
\text { - Post-graduate }\end{array}$ & $\begin{array}{c}46 \\
49 \\
5\end{array}$ & $\begin{array}{c}46.0 \\
49.0 \\
5.0\end{array}$ \\
\hline $\begin{array}{l}\text { Occupation } \\
\text { - } \quad \text { Nursing staff } \\
\text { - } \quad \text { Head nursing }\end{array}$ & $\begin{array}{l}71 \\
29\end{array}$ & $\begin{array}{l}71.0 \\
29.0\end{array}$ \\
\hline $\begin{array}{l}\text { Experience (in years) } \\
\text { - }<5 \\
\text { - } 5-<10 \\
\text { - } \geq 10\end{array}$ & $\begin{array}{l}27 \\
28 \\
45\end{array}$ & $\begin{array}{l}27.0 \\
28.0 \\
45.0\end{array}$ \\
\hline $\begin{array}{c}\text { Range } \\
\text { Mean } \pm \text { SD }\end{array}$ & & \\
\hline $\begin{array}{l}\text { Previous training about central line } \\
\text { related blood stream infection } \\
\text { - No } \\
\text { - Yes }\end{array}$ & $\begin{array}{l}79 \\
21\end{array}$ & $\begin{array}{l}79.0 \\
21.0\end{array}$ \\
\hline $\begin{array}{l}\text { Duration of training program } \\
\text { - None } \\
\text { - }<2 \text { weeks }\end{array}$ & $\begin{array}{l}79 \\
21\end{array}$ & $\begin{array}{l}79.0 \\
21.0\end{array}$ \\
\hline
\end{tabular}




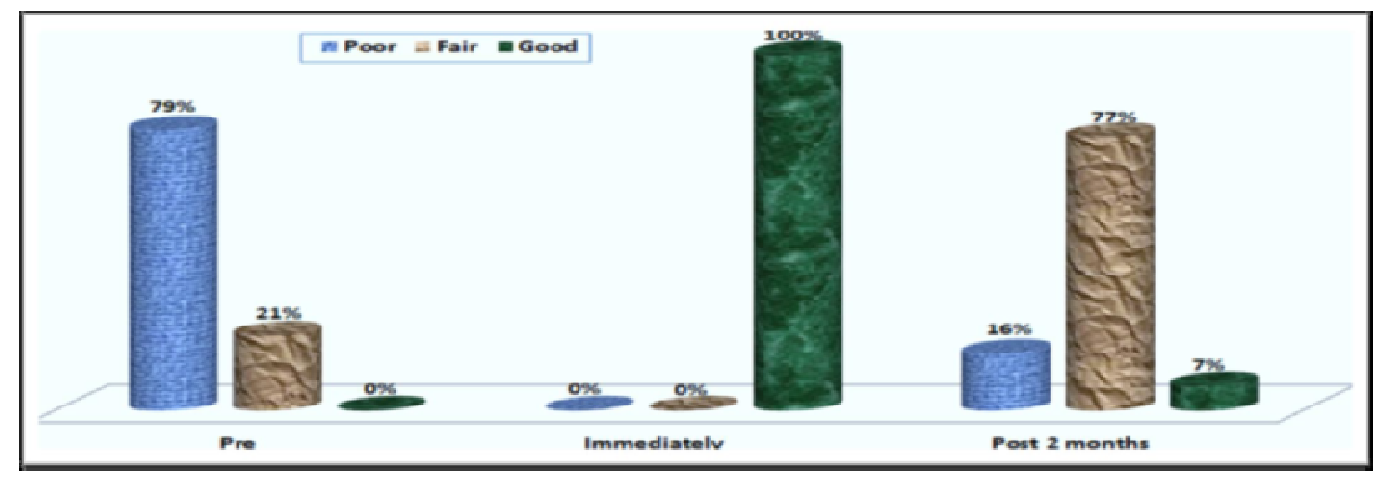

Figure (1): Distribution of the studied nurses according to their total knowledge level regarding $\mathrm{CVC}$ throughout all periods of implementation of nursing educational guidelines

Table (2): Distribution of the studied nurses according to their knowledge domains regarding CVC throughout all periods of implementation of nursing educational guidelines

\begin{tabular}{|c|c|c|c|c|c|c|c|c|c|c|c|c|c|c|c|c|c|c|c|}
\hline \multirow{4}{*}{$\begin{array}{l}\text { Knowledge } \\
\text { domains }\end{array}$} & \multicolumn{18}{|c|}{ The studied nurses $(\mathrm{n}=100)$} & \multirow{4}{*}{$\begin{array}{l}\chi^{2} \\
\mathbf{P}\end{array}$} \\
\hline & \multicolumn{6}{|c|}{ Pre } & \multicolumn{6}{|c|}{ Immediately } & \multicolumn{6}{|c|}{ Post 2 months } & \\
\hline & \multicolumn{2}{|c|}{ Poor } & \multicolumn{2}{|c|}{ Fair } & \multicolumn{2}{|c|}{ Good } & \multicolumn{2}{|c|}{ Poor } & \multicolumn{2}{|c|}{ Fair } & \multicolumn{2}{|c|}{ Good } & \multicolumn{2}{|c|}{ Poor } & \multicolumn{2}{|c|}{ Fair } & \multicolumn{2}{|c|}{ Good } & \\
\hline & $\mathrm{n}$ & $\%$ & $\mathrm{n}$ & $\%$ & $\mathrm{n}$ & $\%$ & $\mathrm{~N}$ & $\%$ & $\mathrm{n}$ & $\%$ & $\mathrm{~N}$ & $\%$ & $\mathrm{n}$ & $\%$ & $\mathrm{n}$ & $\%$ & $\mathrm{n}$ & $\%$ & \\
\hline $\begin{array}{l}\text { 1. Knowledge } \\
\text { regarding CVC }\end{array}$ & 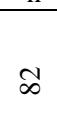 & $\begin{array}{l}\stackrel{8}{1} \\
\text { i }\end{array}$ & $\cong$ & $\begin{array}{l}8 \\
\infty \\
\infty\end{array}$ & 0 & 8 & 0 & $\stackrel{8}{8}$ & 0 & 8 & 8 & $\stackrel{8}{8}$ & 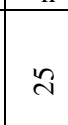 & $\begin{array}{l}8 \\
\ddot{i}\end{array}$ & 닌 & $\begin{array}{l}8 \\
\stackrel{i}{0}\end{array}$ & g & $\begin{array}{l}8 \\
\stackrel{9}{+}\end{array}$ & $\begin{array}{l}272.53 \\
0.000 *\end{array}$ \\
\hline $\begin{array}{c}\text { Range } \\
\text { Mean } \pm \text { SD }\end{array}$ & \multicolumn{6}{|c|}{$\begin{array}{c}(8-19) \\
13.39 \pm 2.84\end{array}$} & \multicolumn{6}{|c|}{$\begin{array}{c}(24-26) \\
25.62 \pm 0.63\end{array}$} & \multicolumn{6}{|c|}{$\begin{array}{c}(5-25) \\
17.47 \pm 3.71\end{array}$} & $\begin{array}{l}F=523.86 \\
P=0.000 *\end{array}$ \\
\hline $\begin{array}{l}\text { 2. Knowledge about } \\
\text { technical } \\
\text { description, site and } \\
\text { care of CVC }\end{array}$ & ๙ & $\begin{array}{l}8 \\
\substack{i \\
\infty}\end{array}$ & $\Xi$ & $\begin{array}{l}8 \\
\stackrel{8}{ \pm}\end{array}$ & $\forall$ & $\underset{8}{8}$ & 0 & 8 & 0 & $\stackrel{8}{8}$ & 8 & $\stackrel{8}{8}$ & 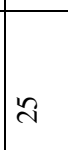 & $\begin{array}{l}8 \\
\ddot{2}\end{array}$ & $\theta$ & $\begin{array}{l}8 \\
\ddot{\lambda}\end{array}$ & in & $\begin{array}{l}8 \\
\text { in }\end{array}$ & $\begin{array}{l}286.95 \\
0.000 *\end{array}$ \\
\hline $\begin{array}{c}\text { Range } \\
\text { Mean } \pm \text { SD }\end{array}$ & \multicolumn{6}{|c|}{$\begin{array}{c}(10-23) \\
14.11 \pm 2.86\end{array}$} & \multicolumn{6}{|c|}{$\begin{array}{c}(26-28) \\
27.30 \pm 0.73\end{array}$} & \multicolumn{6}{|c|}{$\begin{array}{c}(9-26) \\
18.57 \pm 3.54\end{array}$} & $\begin{array}{l}F=635.62 \\
P=0.000 *\end{array}$ \\
\hline \multicolumn{20}{|l|}{\begin{tabular}{l|} 
3. Knowledge about \\
CLABSI
\end{tabular}} \\
\hline $\begin{array}{l}\text { a) Definition, causes, } \\
\text { types, ... }\end{array}$ & 8 & $\stackrel{0}{8}$ & $\stackrel{\infty}{\sim}$ & 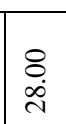 & $m$ & $\begin{array}{l}8 \\
\dot{r}\end{array}$ & 0 & 8 & 0 & 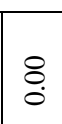 & 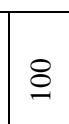 & $\stackrel{0}{8}$ & $\ddot{n}$ & $\begin{array}{l}8 \\
\ddot{1}\end{array}$ & 2 & $\begin{array}{l}8 \\
\stackrel{0}{0}\end{array}$ & in & 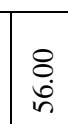 & \begin{tabular}{|l|}
266.89 \\
$0.000 *$
\end{tabular} \\
\hline $\begin{array}{c}\text { Range } \\
\text { Mean } \pm \text { SD }\end{array}$ & \multicolumn{6}{|c|}{$\begin{array}{c}(7-17) \\
10.92 \pm 2.19\end{array}$} & \multicolumn{6}{|c|}{$\begin{array}{c}(17-20) \\
19.56 \pm 0.71\end{array}$} & \multicolumn{6}{|c|}{$\begin{array}{c}(8-18) \\
13.34 \pm 2.49\end{array}$} & $\begin{array}{l}\mathrm{F}=518.26 \\
\mathrm{P}=0.000 *\end{array}$ \\
\hline $\begin{array}{l}\text { b) Assessment, } \\
\text { prevention and care } \\
\text { of CVC }\end{array}$ & 2 & $\stackrel{0}{\circ}$ & オ & $\begin{array}{l}8 \\
\dot{+}\end{array}$ & 0 & $\stackrel{8}{\circ}$ & 0 & $\stackrel{8}{\circ}$ & 0 & $\stackrel{8}{\circ}$ & 8 & $\stackrel{\circ}{8}$ & 离 & $\begin{array}{l}8 \\
\dot{+}\end{array}$ & $n$ & $\begin{array}{l}8 \\
\ddot{n} \\
i n\end{array}$ & $\vec{\lambda}$ & $\stackrel{\circ}{i}$ & $\begin{array}{l}286.17 \\
0.000 *\end{array}$ \\
\hline $\begin{array}{c}\text { Range } \\
\text { Mean } \pm \text { SD }\end{array}$ & \multicolumn{6}{|c|}{$\begin{array}{c}(12-27) \\
20.16 \pm 3.19\end{array}$} & \multicolumn{6}{|c|}{$\begin{array}{c}(36-38) \\
37.59 \pm 0.63\end{array}$} & \multicolumn{6}{|c|}{$\begin{array}{c}(16-34) \\
25.30 \pm 3.82\end{array}$} & $\begin{array}{l}F=968.94 \\
P=0.000 *\end{array}$ \\
\hline $\begin{array}{l}\text { Total knowledge } \\
\text { level }\end{array}$ & 2 & ஜ̊ & $\bar{\sim}$ & 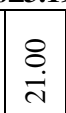 & 0 & $\stackrel{8}{0}$ & 0 & $\stackrel{8}{0}$ & 0 & $\stackrel{8}{\circ}$ & 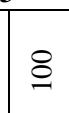 & $\stackrel{0}{8}$ & 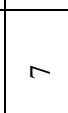 & $\underset{i}{8}$ & $\curvearrowright$ & $\underset{8}{\stackrel{8}{\circ}}$ & 느 & $\stackrel{0}{0}$ & \begin{tabular}{|l|}
381.916 \\
$0.000 *$
\end{tabular} \\
\hline $\begin{array}{l}\text { Range } \\
\text { Mean } \pm \text { SD }\end{array}$ & \multicolumn{6}{|c|}{$\begin{array}{c}(38-74) \\
58.58 \pm 8.31\end{array}$} & \multicolumn{6}{|c|}{$\begin{array}{c}(108-112) \\
110.07 \pm 1.18\end{array}$} & \multicolumn{6}{|c|}{$\begin{array}{c}(51-96) \\
74.68 \pm 7.69\end{array}$} & $\begin{array}{l}F=1604.02 \\
P=0.000 *\end{array}$ \\
\hline $\begin{array}{l}<60 \% \text { Poor } \\
* \text { Significant at level } \\
\text { CLABSI: central line }\end{array}$ & $<0.05$ & $(60-7$ & 75)\% & $\begin{array}{l}\text { Fair } \\
\text { C.V. }\end{array}$ & & G & $\begin{array}{l}\text { ood } \\
\text { venol }\end{array}$ & $\begin{array}{l}75 \% \\
\text { cath }\end{array}$ & neter & & & & & & & & & & \\
\hline
\end{tabular}


Table (3): Distribution of the studied nurses according to their main practice domains levels throughout all periods of implementation of nursing educational guidelines

\begin{tabular}{|c|c|c|c|c|c|c|c|c|c|c|c|c|c|}
\hline & \multicolumn{4}{|c|}{ Pre } & \multicolumn{4}{|c|}{ Immediately } & \multicolumn{4}{|c|}{ Post 2 Months } & \\
\hline & \multicolumn{2}{|c|}{ Unsatisfactory } & \multicolumn{2}{|c|}{ Satisfactory } & \multicolumn{2}{|c|}{ Unsatisfactory } & \multicolumn{2}{|c|}{ Satisfactory } & \multicolumn{2}{|c|}{ Unsatisfactory } & \multicolumn{2}{|c|}{ Satisfactory } & \\
\hline & $\mathrm{n}$ & $\%$ & $\mathrm{n}$ & $\%$ & $\mathbf{n}$ & $\%$ & $\mathbf{N}$ & $\%$ & $\mathbf{N}$ & $\%$ & n & $\%$ & \\
\hline $\begin{array}{c}\text { A. } \\
\text { Practice } \\
\text { regarding } \\
\text { CVC } \\
\text { procedure }\end{array}$ & 80 & 80.00 & 20 & 20 & 0 & 0.00 & 100 & 100.00 & 40 & 40.00 & 60 & 60.00 & $\begin{array}{c}\text { 33.33 } \\
\mathbf{0 . 0 0 0}^{*}\end{array}$ \\
\hline Range & \multicolumn{4}{|c|}{$\frac{1}{(41-80)}$} & \multicolumn{4}{|c|}{$\frac{1}{(90-99)}$} & \multicolumn{4}{|c|}{$\frac{1}{(49-85)}$} & 646.68 \\
\hline Mean \pm SD & \multicolumn{4}{|c|}{$61.16 \pm 9.39$} & \multicolumn{4}{|c|}{$95.50 \pm 2.03$} & \multicolumn{4}{|c|}{$71.41 \pm 7.19$} & $0.000 *$ \\
\hline $\begin{array}{c}\text { B. } \\
\text { Practice } \\
\text { regarding } \\
\text { infection } \\
\text { control } \\
\text { measures }\end{array}$ & 81 & 81.00 & 19 & 19 & 0 & 0.00 & 100 & 100.00 & 0 & 0.00 & 100 & 100.00 & $\begin{array}{c}221.9 \\
1 \\
0.000 *\end{array}$ \\
\hline Range & \multicolumn{4}{|c|}{ (21-45) } & \multicolumn{4}{|c|}{$(54-58)$} & \multicolumn{4}{|c|}{$\frac{1}{(42-56)}$} & 584.45 \\
\hline Mean \pm SD & \multicolumn{4}{|c|}{ 33.37 \pm 7.42} & \multicolumn{4}{|c|}{$56.32 \pm 1.28$} & \multicolumn{4}{|c|}{$47.09 \pm 3.46$} & $0.000^{*}$ \\
\hline $\begin{array}{c}\text { Total } \\
\text { Practice } \\
\text { level }\end{array}$ & 80 & 80.00 & 20 & 20 & 0 & 0.00 & 100 & 100.00 & 11 & 11.00 & 89 & 89.00 & $\begin{array}{l}177.95 \\
0.000 *\end{array}$ \\
\hline
\end{tabular}
$<70 \%$ Unsatisfactory
$\geq 70 \%$ Satisfactory
CVC: central venous
catheter

* Significant at level $\mathrm{P}<0.05$ 
Table (4): Correlation between total practice domains and total knowledge domains among the studied nurses throughout all periods of implementation of nursing educational guidelines

\begin{tabular}{|c|c|c|c|c|c|c|c|c|c|c|c|c|c|c|c|c|c|c|}
\hline \multirow{4}{*}{$\begin{array}{l}\text { Knowledge } \\
\text { domains }\end{array}$} & \multicolumn{18}{|c|}{ Practice domains } \\
\hline & \multicolumn{6}{|c|}{$\begin{array}{l}\text { A. Practice regarding central venous } \\
\text { catheter procedure }\end{array}$} & \multicolumn{6}{|c|}{$\begin{array}{l}\text { B. Practice regarding infection control } \\
\text { measures }\end{array}$} & \multicolumn{6}{|c|}{ Total practice score } \\
\hline & \multicolumn{2}{|c|}{ Pre } & \multicolumn{2}{|c|}{$\begin{array}{c}\text { Immedi } \\
\text { ately }\end{array}$} & \multicolumn{2}{|c|}{$\begin{array}{c}\text { Post } 2 \\
\text { Months }\end{array}$} & \multicolumn{2}{|c|}{ Pre } & \multicolumn{2}{|c|}{$\begin{array}{c}\text { Immedia } \\
\text { tely }\end{array}$} & \multicolumn{2}{|c|}{$\begin{array}{c}\text { Post } 2 \\
\text { Months }\end{array}$} & \multicolumn{2}{|c|}{ Pre } & \multicolumn{2}{|c|}{$\begin{array}{c}\text { Immed } \\
\text { iately }\end{array}$} & \multicolumn{2}{|c|}{$\begin{array}{r}\text { Post } 2 \\
\text { months }\end{array}$} \\
\hline & $\mathbf{r}$ & $\mathbf{P}$ & $\mathbf{R}$ & $\mathbf{P}$ & $\mathbf{R}$ & $\mathbf{P}$ & $\mathbf{r}$ & $\mathbf{P}$ & $\mathbf{R}$ & $\mathbf{P}$ & $\mathbf{r}$ & $\mathbf{P}$ & $\mathbf{R}$ & $\mathbf{p}$ & $\mathbf{r}$ & $\mathbf{p}$ & $\mathbf{R}$ & $\mathbf{p}$ \\
\hline $\begin{array}{l}\text { 1. Anatomy } \\
\text { of the vein }\end{array}$ & $\begin{array}{l}\infty \\
0 \\
0 \\
\\
1\end{array}$ & $\begin{array}{l}+ \\
\stackrel{+}{0} \\
\stackrel{0}{0}\end{array}$ & 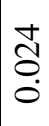 & 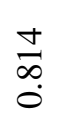 & $\begin{array}{l}\hat{\jmath} \\
\hat{0}\end{array}$ & 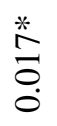 & $\begin{array}{l}\stackrel{0}{0} \\
\text { o. } \\
0\end{array}$ & $\underset{\mathbb{0}}{\stackrel{0}{0}}$ & $\frac{n}{0}$ & $\frac{⿱}{ \pm}$ & $\begin{array}{l}\text { O̦ } \\
0\end{array}$ & $\begin{array}{l}\infty \\
\infty \\
\infty \\
0\end{array}$ & $\begin{array}{l}n \\
0 \\
0 \\
1\end{array}$ & $\stackrel{\sigma}{\sigma}$ & $\begin{array}{l}n \\
0 \\
0\end{array}$ & $\vec{\sigma}$ & $\frac{0}{0}$ & $\begin{array}{l}\text { \& } \\
\text { ठ } \\
0\end{array}$ \\
\hline $\begin{array}{l}\text { 2.Technical } \\
\text { description }\end{array}$ & $\bar{F}$ & $\stackrel{\infty}{\hat{\jmath}}$ & $\stackrel{m}{0}$ & $\stackrel{2}{\circ}$ & $\begin{array}{l}0 \\
0 \\
0 \\
0\end{array}$ & $\stackrel{n}{\tilde{n}}$ & $\begin{array}{l}\text { } \\
8 \\
\dot{0}\end{array}$ & مa & $\stackrel{\infty}{\substack{n \\
i}}$ & \begin{tabular}{l}
$*$ \\
$*$ \\
\multirow{*}{*}{} \\
8 \\
0 \\
0
\end{tabular} & $\stackrel{\infty}{\stackrel{0}{0}}$ & $\begin{array}{l}* \\
\infty \\
\infty \\
0 \\
0\end{array}$ & 官 & $\stackrel{\ominus}{\circ}$ & 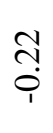 & 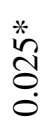 & $\frac{n}{0}$ & $\stackrel{\infty}{\stackrel{\infty}{0}}$ \\
\hline $\begin{array}{l}\text { 3. CLABSI: } \\
\text { Definition, } \\
\text { causes, } \\
\text { types,... }\end{array}$ & $\stackrel{0}{+}$ & $\stackrel{\infty}{m}$ & 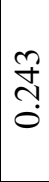 & $\begin{array}{l}\stackrel{*}{*} \\
\stackrel{n}{0} \\
\stackrel{0}{0}\end{array}$ & $\frac{0}{1}$ & $\stackrel{8}{\circ}$ & $\begin{array}{l}\text { ठे } \\
\text { ஸे } \\
\text { iे }\end{array}$ & $\begin{array}{l}\stackrel{*}{\hat{\delta}} \\
\stackrel{0}{0}\end{array}$ & $\stackrel{m}{=}$ & $\underset{\substack{0 \\
0}}{n}$ & $\frac{n}{0}$ & $\stackrel{\text { I }}{\stackrel{0}{0}}$ & $\frac{1}{0}$ & $\stackrel{\infty}{\circ}$ & $\stackrel{\overbrace{}}{0}$ & 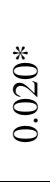 & $\frac{a}{0}$ & $\begin{array}{l}\widetilde{\sigma} \\
\text { ర్ }\end{array}$ \\
\hline $\begin{array}{c}\text { 4. CLABSI: } \\
\text { Assessment, } \\
\text { prevention, .. }\end{array}$ & $\begin{array}{l}0 \\
0 \\
0\end{array}$ & $\begin{array}{l}0 \\
\text { oे } \\
0\end{array}$ & $\frac{n}{2}$ & 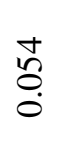 & $\begin{array}{l}\infty \\
0 \\
0 \\
1\end{array}$ & $\stackrel{3}{\frac{3}{0}}$ & $\begin{array}{l}\tilde{n} \\
0 \\
0\end{array}$ & $\begin{array}{l}\text { * } \\
\text { \% } \\
0 \\
0\end{array}$ & $\frac{D}{0}$ & $\begin{array}{l}\stackrel{0}{0} \\
\stackrel{0}{0}\end{array}$ & $\begin{array}{l}n \\
0 \\
0\end{array}$ & $\begin{array}{l}\bar{\sigma} \\
\text { ○े }\end{array}$ & $\hat{n}$ & 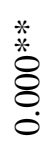 & 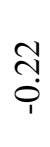 & 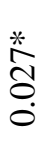 & $\begin{array}{l}\infty \\
0 \\
0 \\
\end{array}$ & $\underset{\sim}{\stackrel{f}{f}}$ \\
\hline $\begin{array}{c}\text { Total } \\
\text { knowledge } \\
\text { score }\end{array}$ & $\begin{array}{l}0 \\
0 \\
1\end{array}$ & $\frac{\hat{\theta}}{0}$ & 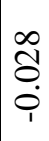 & $\frac{n}{\infty}$ & $\begin{array}{l}\overline{0} \\
0 \\
0\end{array}$ & $\stackrel{\infty}{\dot{H}^{n}}$ & 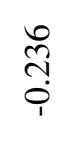 & $\stackrel{*}{\infty}$ & ָ̦ & \begin{tabular}{l}
$*$ \\
$*$ \\
\multirow{2}{*}{} \\
0 \\
0
\end{tabular} & $\frac{1}{0}$ & 市 & $\tilde{\jmath}$ & $\begin{array}{l}\stackrel{*}{2} \\
\text { O̊ } \\
0\end{array}$ & $\frac{n}{0}$ & $\frac{\stackrel{9}{ \pm}}{0}$ & $\begin{array}{l}\tilde{\sigma} \\
\text { ô. }\end{array}$ & $\begin{array}{l}n \\
0 \\
0 \\
0\end{array}$ \\
\hline
\end{tabular}

* Significant at level $\mathrm{P}<0.05$

CLABSI: central line associated blood stream infection

** Highly significant at level $\mathrm{P}<0.01$ 
Table (5): Correlation between socio-demographic characteristics and total knowledge score among the studied nurses throughout all periods of implementation of nursing educational guidelines

\begin{tabular}{|c|c|c|c|c|c|c|}
\hline \multirow{3}{*}{$\begin{array}{l}\text { Characteristics of the } \\
\text { nurses }\end{array}$} & \multicolumn{6}{|c|}{$\begin{array}{l}\text { The studied nurses }(\mathrm{n}=100) \\
\text { Total knowledge score }\end{array}$} \\
\hline & \multicolumn{2}{|l|}{ Pre } & \multicolumn{2}{|c|}{ Immediately } & \multicolumn{2}{|c|}{$\begin{array}{l}\text { Post } 2 \\
\text { Months }\end{array}$} \\
\hline & $\mathbf{R}$ & $\mathbf{P}$ & $\mathbf{r}$ & $\mathbf{P}$ & $\mathbf{R}$ & $\mathbf{P}$ \\
\hline Age (in years) & -0.077 & 0.445 & -0.043 & 0.669 & -0.031 & 0.763 \\
\hline Gender & 0.031 & 0.762 & -0.015 & 0.883 & 0.104 & 0.304 \\
\hline Occupation & -0.341 & $0.001 * *$ & -0.038 & 0.706 & 0.023 & 0.821 \\
\hline Experience (in years) & 0.135 & 0.180 & -0.045 & 0.660 & -0.060 & 0.553 \\
\hline
\end{tabular}

* Significant at level $\mathrm{P}<0.05$

** Highly significant at level $\mathrm{P}<0.01$

Table (6): Correlation between socio-demographic characteristics and total practice score of among the studied nurses throughout all periods of implementation of nursing educational guidelines

\begin{tabular}{|c|c|c|c|c|c|c|}
\hline \multirow{3}{*}{$\begin{array}{l}\text { Characteristics of the } \\
\text { nurses }\end{array}$} & \multicolumn{6}{|c|}{$\begin{array}{l}\text { The studied nurses }(\mathrm{n}=100) \\
\text { Total practice score }\end{array}$} \\
\hline & \multicolumn{2}{|l|}{ Pre } & \multicolumn{2}{|c|}{ Immediately } & \multicolumn{2}{|c|}{$\begin{array}{l}\text { Post } 2 \\
\text { Months }\end{array}$} \\
\hline & $\mathbf{R}$ & $\mathbf{P}$ & $\mathbf{r}$ & $\mathbf{P}$ & $\mathbf{R}$ & $\mathbf{P}$ \\
\hline Age (in years) & -0.015 & 0.886 & -0.213 & 0.033* & 0.059 & 0.560 \\
\hline Gender & 0.121 & 0.231 & 0.124 & 0.221 & -0.058 & 0.568 \\
\hline Occupation & 0.044 & 0.664 & -0.104 & 0.304 & 0.023 & 0.821 \\
\hline Experience (in years) & 0.038 & 0.708 & 0.181 & 0.072 & 0.072 & 0.474 \\
\hline
\end{tabular}

* Significant at level $\mathrm{P}<0.05$

** Highly significant at level $\mathrm{P}<0.01$ 


\section{Discussion}

Nurses in intensive care units have the most direct and continuous role in performing care maintenance of the central venous catheter insertion site procedure and they should be experienced with supportive care measures in the insertion and maintenance of central lines. Therefore must be prepared to be able to contribute to the primary prevention guidelines of central line associated blood stream infection through improved their knowledge and practice toward proper use of $\mathrm{CVC}$ to minimize the risk of complications and improve patient's quality of life. So this is study was conducted to evaluate the effect of educational guidelines on nurse's knowledge and practice regarding central line associated blood stream infection at intensive care unit ${ }^{(33,34)}$.

\section{Concerning tosocio-demographic}

characteristics of the studied nurses, the

results of the present study showed that nearly more than half of the nurses were aged from 20 to less than 31 years old. This may reflects the demanding nature of critical care unites service, so that older nurses may find it difficult to cope with the load of work required and prefer the newly graduate to work in the critical care units, as they had the ability to acquire knowledge and change their behaviors

based on submission of up to date knowledge. This finding was in the same line with Elbilgahy et al. (2019) ${ }^{(35)}$ who reported that nearly half of the studied nurses who are worked in intensive care units were aged from 20 to less than 30 years old. Moreover, Abbady and Gaballah (2019) (36) reported that the mean age of the studied nurses who are caring for central line in intensive care unit was $27.02 \pm 2.15$. On the other hand, this finding was consistant with Esposito and Guillari (2017) ${ }^{(37)}$ who reported that nearly three-quarters of nurses who are caring for patients with central venous catheter were aged from $(20<40)$. The finding parallel to Bayoumi and Mahmoud (2017) ${ }^{(38)}$ who reported that three quarter of the nurses who are deal with central line in hemodialysis unit were less than 25 years old. Additionallythis finding disagrees with Mishras (2016) ${ }^{(39)}$ who reported that the most of the studied nurses in intensive care unit were aged from $(29<39)$.

\section{Concerning to sex and marital status,} the result of the present study showed that the majority of the studied nurses were females and married. This finding was in the same line with Shah (2019) ${ }^{(40)}$ and Raghep and $\operatorname{Elgazar}(2019)^{(41)}$ who 
reported that the majority of the studied nurses who working in intensive care unit and deal with central venous access device were females and nearly three quarters of them were married

In relation to nurses' educational leveland occupation, it was seen that nearly half of the studied nurses had bachelor degree of nursing. It could be due to hospital policy as they prefer highly qualified nurses in the critical care unites rather than other graduate to be able to carry up their responsibility. This finding was in the same line with Sami et al.(2018) $^{(42)}$ and Muslim et al. (2018) ${ }^{(43)}$ who reported that most of the studied nurses who providing care for patients with central line were had bachelor of nursing.On the other hand, this finding disagreement with Moursy and Sharaf(2017) (44), who study bout "vascular access care at hemodialysis unit; nurses' compliance to infection prevention and control practice", who reported that more than two third of the studied nurses the educational level are diploma.

Concerning to nurses' years of experiences, the present study results showed that nearly half of the studied nurses had ten or more years of nursing experience. This finding was congruent with Deshmukh et al. (2014) $^{(45)}$ who conducted that study about vascular access care among hemodialysis patients and mentioned that nearly of the studied nurses who were providing care to patients had more than twenty years of experiences. On the other hand, this finding was disagreement with Elbilgahy et al. (2019) (35) who stated that approximately two thirds of the studied nurses who work in hemodialysis units to care for central venous catheter having 10 years of experience.

\section{According to nurses' previous training} about central line related blood stream infection, nearly four fifths of the studied nurses did not have previous training about central line related blood stream infection. This finding was in the same line withAbdelsatir (2013) ${ }^{(46)}$ and Hawkins (2018) ${ }^{(47)}$ who conducted study about central line and infection control measures and found that the majority of the studied nurses didn't attend any pervious training about central line.Converse toMoursy andSharaf(2017) ${ }^{(44)}$, reported that the majority of the studied nurses attended infection prevention training programs about Central line during work in the critical care unit.

Concerning to level of knowledge of the studied nurses regarding central venous catheter, central line associated blood stream infection and its management pre, immediately and post 2 months of 
implementation of nursing educational guidelines, the results of the present study showed that, there was statistical significant improvement in the level of nurses' knowledge regarding central venous catheter, central line associated blood stream infection and its management. Where the majority of the studied nurses had poor level of knowledge pre nursing educational guidelines, whereas all of them had good level of knowledge after implementation of nursing educational guidelines.

The poor level of nurses' knowledge about central line and central line associated blood stream infection pre nursing educational guidelines may be related to lack of training program that must be conducted to improve nurses' knowledge and they depending on acquiring their knowledge from the experience of their colleagues. The implementation of nursing educational guidelines has a positive effect on nurses' knowledge as the majority of the studied nurses had good level of knowledge post nursing educational guidelines. This improvement in knowledge indicated effectiveness of the nursing educational guidelines and the teaching sessions that were done by the researcher. Also, this improvement may be related to nurses' desire to acquire new knowledge, active participation and regular attendance in the sessions of the program that was reflected on knowledge scores of the studied nurses.

This finding was consistent with Raghep and Elgazar(2020) ${ }^{(41)}$ who revealed that the majority of the studied nurse's had unsatisfactory level of knowledge pre educational program intervention and reached to satisfactory level immediate and post program regarding care of patients during insertion of central venous catheter. In addition, this result was supported by Fayed et al. (2016) ${ }^{(48)}$ who illustrated that the lack of continuous education and training programs about central line led to poor level of nurses' knowledge preprogram implementation, while all of them had good knowledge after program implementation. Additionally, the finding of the present study was in the same line with Sakshi (2019) ${ }^{(49)}$ who reported that the implementation of educational program regarding care of central venous catheter led to statistical significant improvement of knowledge level among nurses who were working in intensive care unit.

This finding was consists with Aloush (2018) $)^{(50)}$ who reported that the majority of nurses had poor level of knowledge pre educational guidelines about procedure of central line. In addition the finding of the present study was in the same line with Al Qadire (2017) ${ }^{(51)}$ who revealed that 
nurses of intensive care units had insufficient knowledge about central line associated blood stream infection.

Also, this result was supported byVenkatesan et al. $2018^{(52)}$ who reported that all nurses who were providing care for patients with central line in intensive care unit had satisfactory level of knowledge post educational program regarding prevention of central line associated blood stream infection .In addition to ,this result agreed with Chen et al. (2015) ${ }^{(53)}$ who revealed that there was statistical improvement of knowledge scores among nurses who were providing care for patients with central line in intensive care units post completion of educational guidelines.

Concerning to level of practice of the studied nurses regardingcentral venous catheter procedures and infection control measures, the present study showed that there was a statistical significant improvement in the level of nurses' practice regarding both central venous catheter and infection control measures, where the most of studied nurses had unsatisfactory level of practice pre nursing educational guidelines whereas all of them were had satisfactory level of practice immediately after implementing nursing educational guidelines .
The unsatisfactory level of nurses' practice regarding central venous catheter and infection control measures pre educational guidelines may be related to inadequate level of nurses' knowledge pre guidelines, shortage of nursing staff, lack of updating knowledge, lack of continuous education and deficiency of in-service training program. The implementation of educational guidelines has a positive effect on nurses' practice as the majority of studied nurses had satisfactory level of practice post educational guidelines. This improvement indicates effectiveness of the nursing guidelines that led to enhancement of nurses' knowledge which reflected on nurses' practice.

This finding was in the same line with Bayoumi (2017) ${ }^{(38)}$ who reported that there was a highly statistical significant difference between nurses' practice pre and post implementation of educational guidelines as the majority of nurses had adequate level of practice post implementing the nursing educational program regarding central line.

Additionally, this finding was in the same line with Yousifet al.(2017) $)^{(54)}$ who reported that there was a highly statistical improvement in the level of nurse' practice regarding infection control measures of central line care where about more than half of the studied nurses were had in 
competent practice at the pre-educational guidelines .While more than three quarter of them had a competent practice at post intervention phase.

Moreover, this finding was the same line with Tang et al. (2014) ${ }^{(55)}$ who reported that there was highly significant improvement in the level of nurse' practice regarding central line associated blood stream infection after implementation of infection control bundle. This finding was supported with Sacks et al. (2014) ${ }^{(56)}$ who reported that majority of the studied had low level of practice regarding care of patients with central line in pre intervention, while the majority of them had good level of practice after nursing intervention.

Also, this finding was supported with Kun et al.(2017) ${ }^{(57)}$ who reported that there was a highly statistical improvement in the level of nurses practice regarding care of peripherally inserted central catheter after and catheter maintenance after educational program.

Moreover, this finding was the same line with Wright et al. (2013) ${ }^{(58)}$ who reported that there was a highly statistical improvement in the level of nurses' practice regarding disinfection of catheter hubs and prevention of central line associated blood stream infection.
Concerning correlation between both of total knowledge score, total practice score and their domains, findings of the present study reported that there was positive significant correlation between total knowledge score and practice score of the studied nurses pre nursing educational guidelines. It may be related to the nurses poor training can be skilled to unsatisfactory practice. This finding was in the same line with Esposito et al. (2017) ${ }^{(37)}$ who showed thatthere was significant correlation between total knowledge score and practice score of the studied nurses during nursing educational guidelines. Moreover, this finding agreed with ElSolet al. (2017) ${ }^{(59)}$ who conducted a teaching moduleregarding prevention of central-line associated blood stream infection on intensive care unit nurses and reported that implementation of the program that is organized according to the needs of nurses has beneficial effect in improving the nurses knowledge and practice.

Moreover,this finding was agreed with Cooper et al. (2014) ${ }^{(60)}$ who implemented an educational program regarding central line care and confirmed that enhanced nurses' knowledge led to improve nurses' practice. Also, this finding agreed with Awad et al. (2019) ${ }^{(61)}$ Who reported that there was significant correlations between 
total knowledge scores and total practice scores regarding central venous catheter care bundle on critical care nurses at emergency department.

Moreover,this finding was consistent with Caetano et al. (2014) ${ }^{(62)}$ with who found that enhanced nurses' knowledge regarding central venous catheter led to improve nurses' practice regarding prevention of central line complications. In addition, Kadium (2015) ${ }^{(63)}$ who conducted a study in hemodialysis unit and found that there was statistical significant correlations between total knowledge scores and total practice scores regarding central venous catheter care.

\section{Concerning to correlation between} socio-demographic characteristics and total knowledge score among the studied nurses pre, immediately and post 2 months of implementation of nursing educational guidelines, the findings of the present study reported that there was a negative statistical significant correlation between occupation of the studied nurses and total knowledge score pre nursing educational guidelines. It may be related to that, head nurses were poor level of knowledge score due to absence of educational training program about central line and they were occupied with administrative work. This finding was in the same line with Kadium et al. (2015) ${ }^{(63)}$ who clarified that there was a statistical significant correlation between occupation of the studied nurses and total knowledge score pre nursing educational guidelines. In the same line with Yousef (2017) ${ }^{(54)}$ who noticed that nurses' knowledge scores were in significant relation with sociodemographic data of nurses. This finding agreed with Barbosa1 et al. (2017) $)^{(64)}$ who observed that there was a statistical significant correlation between total knowledge score and age and years of experiences post implementation of educational program regarding central line care.

Moreover, this finding was agreed with Koutzavekiaris et al. (2017) ${ }^{(65)}$ who reported that there was negative statistical correlation between age of the studied nurses and total knowledge score pre implementation of educational guidelines. Also, this finding was in the same line with Kokila (2018) ${ }^{(66)}$ who noticed that there was significant correlation between occupation and total knowledge score about central line care in hemodialysis unit.

Concerning correlation between sociodemographic characteristics and total practice score of among the studied nurses pre, immediately and post 2 months of implementation of nursing educational guidelines, the finding of the present study 
revealed that there was a statistical significant correlation between age of the studied nurses and total practice score immediately after implementation of nursing educational guidelines. It may be related to that, the majority of nurses were aged from 20 to 40, this increasing their experiences and consequences lead to satisfactory level of practice regarding central line care and prevention of infection.This finding was in the same line with Caetano et al. (2019) ${ }^{(62)}$ who reported that there was a statistical significant correlation between age of the studied nurses and total practice score pre nursing educational guidelines. This finding agreed with Fayed et al. (2016) ${ }^{(48)}$ who observed that there was a statistical significant correlation between total practice scores and age \&years of experiences post educational guidelines regarding central line In addition,Yousif et al. (2017) ${ }^{(54)}$ who reported that there was a positive correlation between age and total practice score pre implementing nursing educational program about central line associated blood stream infection.

On the other hand, this finding disagreed with Aloush (2018) ${ }^{(50)}$ who noticed that there was no significant correlation between total practice scores and sociodemographic characteristics of the studied nurses throughout the educational program regarding central line.

\section{Conclusion}

\section{Depending on the finding of the present}

study, it was concluded that:

Central venous catheter is a common procedure that is performed in intensive care units for a variety of indications. Central lines are known to be associated with risk of complications as infections and hemorrhage, thus good nurses' knowledge and practice of care are crucial in limiting these risks, also the nurse to be supposed to integrate safe care practice into their care to improve patients outcome (33).

- The implementation of nursing educational guidelines have a positive impact in improving nurses' knowledge and practice regarding prevention of central line associated blood stream infection.

- There was a significant statistical improvement in the nurses' total knowledge and practices mean scores pre, immediately and 2 months post nursing educational guidelines.

- There was a positive significant correlation between total knowledge score \&total practice during and after 2 months of implementation in nursing educational guidelines. 
- There was a statistical significant correlation between sociodemographic characteristics and total knowledge and practice score among the studied nurses throughout all periods of implementation of nursing educational guidelines.

\section{Recommendations}

\section{Depending on the results of the present} study, it was recommended that:

Nurses should attend the seminars and the in-service training programs about central line associated blood stream infection and care for implanted ports for gaining updated knowledge and enhancing their practice.

- The nurses must attend external training courses and conferences to upgrade their knowledge and practices in their field and the evidence based guidelines for CLABSI prevention should be incorporated in all nursing curricula.

- Nurses should use central line insertion checklist to decrease mistakes and avoid infection.

- Nurses should be congruent with infection control guidelines in care of patients with central line.

- Nurses should be aware about pre discharge instructions that are given to the patient with implanted ports to prevent infection.

\section{References}

1. Said N, YassienS,Ameen D. Factors affecting nurses' performance toward central line associated blood stream infection in critical care units. Egyptian Journal of Health Care. 2020; 11(1):234-7.

2. Abdullah M, Mohammed W, Ismail M. Nurses' knowledge and practices about administration of medications via central line among critically ill Patients. Journal of Education and Practice.2014; 5(1): 147-59.

3. Shah H, Schwartz J, Luna G, Cullen D. Bathing with $2 \%$ ChlorhexidineGluconate: Evidence and costs associated with Central Line-Associated Bloodstream Infections. Critical Care Nursing Quarterly. 2016; 39(1): 42-50.

4. Raynak A. Knowledge of central venous access devices among nurses in two acute care facilities in Canada. Vascular Access. 2018; 12(2):7-11.

5. Ajenjo M, Morley J, Russo A, McMullen K, Robinson M ，Warren D. Peripherally inserted central venous catheter-associated bloodstream infections in hospitalized adult patients. Infection Control \& Hospital Epidemiology. 2017; 32(02): 125-30.

6. Centers for Disease Prevention and Control. Blood stream infection event 
(Central Line-Associated Bloodstream Infection and Non-central Line Associated

Bloodstream Infection).2020. Available at: https:// www. cdc. gov/ hai/ progressreport/index.html. Retrieved on $22 / 11 / 2020$.

7. KalenderN,TosunN. Nursing studies about central venous catheter care. International Journal of Caring Sciences. 2015; 8 (2): 461-3.

8. Schallom M, Prentice D,Sona C, Micek S, Skrupky L. Heparin or $0.9 \%$ sodium chloride to maintain central venous catheter patency: A randomized trial. Critical Care Medicine. 2018; 40(6):1820-6.

9. Lopez A. A quality improvement program combining maximal barrier precaution compliance monitoring and daily ChlorhexidineGluconate baths resulting in decreased central line bloodstream infections. Dimensions of Critical Care Nursing. 2011; 30(5): 293-8.

10. Macklin D. Catheter management. Seminars in Oncology Nursing. 2018; 26(2): 113-20.

11. Maki D,Crnich C. Line sepsis in the ICU: Prevention, diagnosis and management. Semin.Respir. Crit. Care Med.2016;24(1):23-36.
12. Blot S, Peleman R,Vandewoude K. Invasive devices: No need? No use! Intensive Care Med. 2007;33(2):20911.

13. Vankoo T, Boer A, Manniën J.Incidence and risk factors of deviceassociated infections and associated mortality at the intensive care in the Dutch surveillance system. Intensive Care Med. 2017;33(2):271-278.

14. Infusion Nurses Society. Infusion nursing standards of practice. Journal of Infusion Nursing.2017; 34(1):11-2.

15. Royal College of Nursing. IV Therapy Forum. Standards for infusion nursing. Royal College of Nursing IV Therapy Forum. 2017;207.

16. Schiffer P, ManguJ, Wade C. Central venous catheter care for the patient with cancer: American Society of Clinical Oncology clinical practice guideline. Journal of Clinical Oncology.2015; 31(10): 1357-60.

17. Goossens G. Totally implantable venous access devices: Malfunction problems.Unpublished Ph.D. Thesis. KU Leuven: Leuven. Belgium;2019.

18. Adhikari S, Blaivas M, Morrison D, Lander L. Comparison of infection rates among ultrasound-guided versus traditionally placed peripheral 
intravenous lines.Ultrasound Med. 2018;29(5):741-7.

19. AlexandrouE, Spencer R, Frost A, Parr J, Davidson M,Hillman M. A review of the nursing role in central venous annulation: Implications for practice policy and research. J Clin.Nurs. 2019;19(3): 1485-94.

20. Alexandrou E,Spencer $T$, Frost $S$, Mifflin N, Davidson P, Hillman K. Central venous catheter placement by advanced practice nurses demonstrates low procedural complication and infection rates-a report from 13 years' service. Crit Care Med. 2014; 42(3):536-43.

21. Moureau N. Vessel Health and Preservation. $2^{\text {nd }}$ Ed. Australia:Springer Pubmed, 2019. 855-67.

22. Pikwer A, Hammarskj F, Larsson A, Lindgren S, LindwallR, Taxbro K, et al. Clinical guidelines on central venous catheterisation. Swedish Society of Anesthesiology and Intensive Care Medicine. ActaAnaesthesiol Scand. 2014; 58(5):508-24.

23. Gallieni M, Martina V, Rizzo $M$, Gravellone L, Mobilia F,Giordano A, et al. Central venous catheters: Legal issues. Journal of Vascular Access. 2017;12(1):273-9.
24. Fairfax L, Christmas A, Norton H,Jacobs D. Breakdown of the consent process at a quaternary medical center: Our full disclosure. Am Surg. 2012;78(1):855-63.

25. Moureau N, Lamperti M, Kelly J, Dawson R, Elbarbary M, Van BoxtelJ , et al. Evidence-based consensus on the insertion of central venous access devices: Definition of minimal requirements for training. Br Journal of Anesthesia. 2013; 110(1):347-56.

26. Nowak J, BrilliR, Lake M. Reducing catheter-associated bloodstream infections in the pediatric intensive care unit: Business case for quality improvement. Pediatric Crit Care. 2010;11(2):579-87.

27. Rosenthal V. Central line-associated bloodstream infections in limitedresource countries: A review of the literature. Clin. Infect Dis. 2019; 49(1):1899- 907.

28. Tille P, Scott S. Diagnostic Microbiology. $13^{\text {th }}$ Ed. New York: Elsevier. 2013.167-70.

29. Chesshyre E, Goff Z. The prevention. diagnosis and management of central venous line infections. Journal of Infection. 2015; 71(2):59- 75.

30. Zimlichman E,Henderson D, Tamir O. Health care-associated infections: A meta-analysis of costs and financial 
impact on the US health care system. JAMA Internal Medicine. 2018:173(22):2039-46.

31. Shore A,Sandoe J. Blood cultures. Student BMJ. 2008; 16(1): 324-325.

32. Gerstman B. Basic Biostatistics, Statistics for Public Health Practice. Jones and BartletPublisher: Canada. 2018.

33. Rupp E, Yu S, Huerta T. Adequate disinfection of a split-septum needleless intravascular connector with a 5-second alcohol scrub. Infect Control Hosp.Epidemiol. 2016; 33(7):661-5.

34. Schallom E, Prentice D, Sona C, Micek T, SkrupkyP. Heparin or 0.9\% sodium chloride to maintain central venous catheter patency: A randomized trial. Crit Care Med. 2018;40(6):1820-6.

35. Elbilgahy A. Evidence-based educational intervention for nurses' about prevention of Central Line associated blood stream infection. Middle East Journal of Nursing.2019; 13(1): 3-16.

36. Abbady A,GaballahS. Bundle of care for improving nurses' performance related to central line associated blood stream infection. American Journal of Nursing Research. 2019; 7(4):46.
37. Esposito R, Guillari A, AngelilloF. Knowledge, attitudes, and practice on the prevention of central lineassociated bloodstream infections among nurses in oncological care: A cross-sectional study in an area of southern Italy. PLoS ONE .2017;12(6):25.

38. Bayoumi M, Mahmoud N. Effect of education program on nurses' knowledge and practice regarding care of central venous line in hemodialysis: Evidence-based practice guidelines. Egyptian Nursing Journal.2017; 14 (2):92-95.

39. Mishras S, Misrar F, Azima E, Baranoia A. Incidence, risk factors and associated mortality of central line-associated bloodstream infections at an intensive care unit in northern India. International Journal for Quality in Health Care. 2017; 29(1):63-67.

40. Shah M, Qasim M. Practice of nursing care for central venous catheter among ICUs nurses in private tertiary care hospital Peshawar. Nurse Health Care.2017; 2(2):3.

41. RaghepM,Elgazar W. Effects of an educational program on the nurses' performance regarding vascular access infection prevention. Egyptian Journal of Health Care.2020; 4(1):13. 
42. Sami M, Faris A. Nurses' compliance with central line associated blood stream infection prevention guidelines: Observational study.Saudi Med J. 2018; 39 (3):277.

43. Muslim S, Qasim M, Zeeshanwahab F. Practice of nursing care for central venous catheter among ICUs nurses in private tertiary care hospital Peshawar, KP. Nurse Health Care.2017;2(2): 3

44. Moursy S, Sharaf E. Vascular access care at hemodialysis unit; nurses' compliance to infection prevention and control practices. Journal of Nursing and Health Science.2017;6(2):61-69.

45. Deshmukh M, Shinde, M. Impact of structured education on knowledge and practice regarding venous access device care among nurses. International Journal of Science and Research (IJSR).2014;3(12): 895-901.

46. Abdelsatir S. Evaluation of nursesawareness and practice of Hemodialysis access care in Khartoum State, Sudan. Arab J Nephrol. Transplant. 2016;6(2):119-21.

47. Hawkins Sh. The effect of individualized nurse report cards and unit case reports on the awareness and attitudes of nurses towards CLABSI contributing factors. . Nurse Health Care. 2018;15(7):26.
48. Fayed N, ElbahnasawyH, Omar T. Effect of instructional program on nurses' compliance with universal precautions of infection control. International Journal of Novel Research in Healthcare and Nursing.2016; 3(1): 81-92. Available at: www.noveltyjournals.com.

49. Sakshi A, Verma M, SalujaV. Effectiveness of educational program regarding central venous catheter care bundle in terms of knowledge and practice of nursing personnel .International Journal of Advanced Research.2019;7(10):1033-35.

50. Aloush S. Educating intensive care unit nurses to use central venous catheter infection prevention guidelines: Effectiveness of an educational course. Journal of Research in Nursing.2018; 23(5):408.

51. Al QadireM. Oncology nurses' knowledge of guidelines for preventing catheter-related bloodstream infections. American Journal of Infection Control. 2017; 45(9): 95-97.

52. Venkatesan J, Manikandan L. Effectiveness of central line bundle care upon the knowledge and compliance staff nurses in the ICU. International Journal of Advance 
Research, Ideas and Innovations in Technology. 2018; 4 (3):2059-63.

53. Chen S, Yao J, Chen J, Liu L, Miu A, Jiang Y. Knowledge of guidelines for the prevention of intravascular catheter-related infections : A survey of intensive care unit nursing staffs in china. International Journal of Nursing Sciences. 2015; 2(4): 383-8.

54. Yousif K, Abu-Aisha H, Abboud O. The effect of an educational program for vascular access care on nurses' knowledge at dialysis centers in Khartoum State, Sudan. Saudi J Kidney Dis Transpl. 2017;28(5):102733.

55. Tang HJ, Lin HL, Lin YH. The impact of central line insertion bundle on central line-associated bloodstream infection. BMC Infect Dis. 2016;14(3):356.

56. Sacks G, DiggsB, Hadjizacharia P, Green D, Salim A, Malinoski D. Reducing the rate of catheterassociated bloodstream infections in a surgical intensive care unit using the Institute for Healthcare Improvement central line bundle, The American Journal of Surgery. 2014; 207(6):81723.

57. Kun C, Yan J, Suwen X, Yan L, Min S. Effect of specialty training on nursing staff's KAP on PICC and catheter maintenance. Biomed Res. 2017; 28 (20):9145-7.

58. Wright M, Tropp J, Schora D, DillonGrant M, Peterson K. Continuous passive disinfection of catheter hubs prevents contamination and bloodstream infection. American Journal of Infection Control.2018; 41 (1): 33-8.

59. El-Sol A, Badawy A. The effect of a designed teaching module regarding prevention of central-Line associated blood stream infection on ICU nurses' knowledge and practice. American Journal of Nursing Science. 2017; 6(1): 11-8.

60. Cooper K, Frampton G, Harris P, Jones J, Cooper T. Are educational interventions to prevent catheterrelated bloodstream infections in intensive care unit cost-effective? Journal of Hospital Infection. 2014;86 (1): 47-52.

61. Awad SH, Elfeky H, Sultan M, Abo Seda A. Critical care nurses' emergency hospital, Mansoura University knowledge and practices regarding central venous line care bundle at emergency hospital. Mansoura Nursing Journal (MNJ), 2019;6(2 ):182.

62. Caetano C, Bueloni T, Ponce D. Interventions for preventing infectious 
complications in Haemodialysis

patients with Central Venous

Catheters. European Medical Journal.

2019; 18(5):98.

63. Kadium MJ. Improving Nurses' Knowledge to Reduce CatheterRelated Bloodstream Infection in Hemodialysis Unit. Walden University Scholarwork.

2015https://scholarworks.waldenu.edu /dissertations/1623

64. Barbosa1 C, Canhestro M, Gonçalves M,Guimarães G. Knowledge of the nursing team on care with central venous catheter. Journal of Nursing.2017;11(11):4345-56.

65. Koutzavekiaris I,Vouloumanou EK, Gourni M, Rafailidis PI, Michalopoulos A , Falagas ME. Knowledge and practices regarding prevention of infections associated with central venous catheters: A survey of intensive care unit medical and nursing staff. Am J Infect Control. 2017;39(1):542-7.

66. Kokila S. A study to assess the effectiveness of structured teaching programme regarding prevention of central line associated blood stream infection in terms of knowledge and skill among staff nurses in selected private hospital at Dharmapuri. Unpublished Master Thesis.
Dharmapuri: SrriPaspo College of Nursing. 2018.195. 